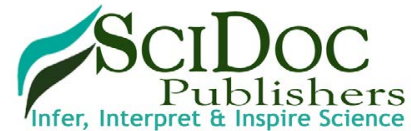

International Journal of Respiratory Disease, Care \& Medicine (IJRDM)

ISSN: 2577-4409

\title{
The Stop-Bang Score and Prediction of Severity of Obstructive Sleep Apnea in Surgical Population
}

\author{
Research Article
}

Kharrat $\mathrm{A}^{1 *}$, Cheikhrouhou $\mathrm{H}^{1}$, Iheb S $\mathrm{S}^{1}$, Rahma $\mathrm{D}^{1}$, Wadii $\mathrm{D}^{1}$, Bouhlel $\mathrm{A}^{1}$, Frikha $\mathrm{M}^{1}$, Msaad $\mathrm{S}^{2}$, Kammoun $\mathrm{S}^{2}$, Karoui $\mathrm{A}^{1}$

${ }^{1}$ Department of Anesthesiology, Habib Bourguiba Hospital, Sfax, Tunisia.

${ }^{2}$ Department of Pneumology, Hedi Chaker Hospital, Sfax, Tunisia.

\section{Abstract}

Background: The "STOP BANG" questionnaire has been developed for screening obstructive sleep apnea syndrome (OSAS) as defined by the apnea/hypopnea index (AHI). It is a common tool used in general population to screen OSAS. We research a correlation between the score of "STOP BANG" questionnaire (0 to 8$)$ and the severity of the OSAS classified into 4 categories ranging from none to severe.

Methods: Demographic and anthropomorphic data, blood pressure and the presence of snoring, tiredness/sleepiness and observed apneas were collected from 150 patients who underwent ventilatory polygraphy. "STOP BANG" questionnaire score was defined for each patient with an ordinal rating of 0 to 8 . Proportional odds logistic regression analysis was conducted to predict severity of sleep apnea based upon the AHI: none (AHI $<5 / H$ ), mild (AHI $\geq 5 / H$ to $<15 / H$ ), moderate $(\geq 15 / \mathrm{H}$ to $<30 / \mathrm{H})$, and severe $(\mathrm{AHI} \geq 30 / \mathrm{H})$.

Results: Linear model $\left(\mathrm{R}^{2}=0.373\right)$ was developed that predicted AHI severity. The linear model showed a progressive increase in the probability of severe (14\% to $85 \%$ ) and progressive decrease in the probability of none (57\% to $43 \%$ ). The probability to have mild, moderate and severe OSAS increases with high scores of "STOP BANG" questionnaire.

Conclusions: The STOP BANG questionnaire may be useful to categorize OSAS severity in surgical population.

Keywords: Stop Bang; Screening; Obstructive Sleep Apnea Syndrome; Prediction; Severity.

\section{Background}

Obstructive sleep apnea syndrome (OSAS) is a disorder a disorder characterized by repetitive periods of apnea and hypopnea during sleep [1]. OSAS has been associated with cardiovascular and neurovascular diseases, metabolic disorders, and neurocognitive dysfunctions [2]. Patients with untreated SOSA have more frequent cardiorespiratory complications in the perioperative environment compared to patients treated prior to surgery [3-5]. Therefore, a simple and reliable screening test is needed to identify patients with OSAS. With increasing numbers of patients suspected of having OSAS, a screening test is also needed in order to prioritize those patients for urgent evaluation and treatment.

Screening tests have been used in order to provide greater accuracy, such as the "STOP BANG" questionnaire [6]. This screening method is compared with a single parameter, apnea/hypopnea in- dex (AHI) derived from polysomnography with is the gold standard for diagnosis of OSAS [7]. Identification of patients with OSAS by "STOP BANG" questionnaire has been based upon a binary decision (absence/presence).

"STOP BANG" questionnaire was validated as an easy and effective screening method to exclude patients with undiagnosed severe sleep apnea from going to surgery $[6,8]$. 8 yes or no responses was delivered, a score higher or equal to 3 was used to suspect OSAS. Because 3 positive responses are considered the same as 8, a "STOP BANG" score higher than 3 indicates that the patient could have mild, moderate or severe OSAS. The STOP BANG questionnaire was chosen for this study because of its simplicity. We hypothesized that the score of the "STOP BANG" questionnaire would correlate with the severity of the OSAS. Giving probabilities of disease severity on 4 levels (none, mild, moderate, and severe) should provide more information than a binary outcome.

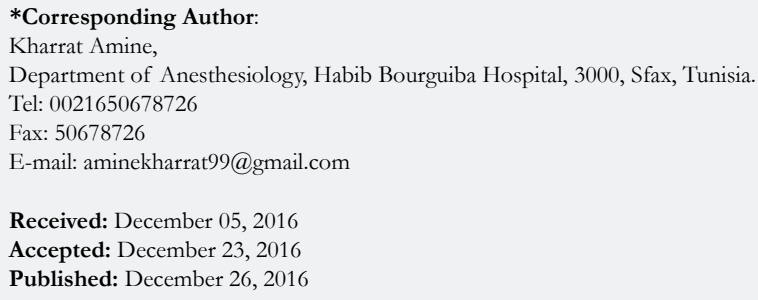

Copyright: Kharrat $\mathbf{A}^{\circ}$ 2016. This is an open-access article distributed under the terms of the Creative Commons Attribution License, which permits unrestricted use, distribution and reproduction in any medium, provided the original author and source are credited. 


\section{Methods}

This is a clinical study, prospective, conducted after approval of the local ethics committee and informed consent of all patients. This study included 150 patients proposed for elective surgery and anesthetic consultation in H. Bourguiba hospital in Sfax in collaboration with the hospital's pulmonology department Hedi Chaker on a 6-month period from 1 November 2011 to 1 May 2012.

The objective of this study was to search a correlation between severity of OSAS and high scores of "STOP BANG" questionnaire. The inclusion criteria for patients: age greater than 18 years; proposed for programmed urologic surgery, general surgery, cardiovascular and thoracic surgery, maxillofacial surgery, otorhinolaryngology surgery, ophthalmology and orthopedic. The exclusion criteria for patients: age less than 18 years; refusal to participate in the study; patient already known suffering from OSAS; severe chronic renal failure, stroke, dementia or Parkinson's syndrome; patients hospitalized for emergency surgery. Exclusion criteria of patients: non-compliance with the study protocol; uncooperative patients and patients lost.

Our study was conducted in two phases: a screening phase to the anesthesia consultation and the registration phase nocturnal ventilation during sleep in pulmonology department.

The first OSA screening phase by the questionnaire "STOP BANG" was held at the anesthesia consultation. During this phase, 150 patients included were informed of the study protocol. For each patient we collected the following data including: age, sex, weight $(\mathrm{kg})$, height $(\mathrm{cm})$ and body mass index (BMI), neck circumference $(\mathrm{cm})$, the main medical and surgical history and habits of patients, the criteria for intubation of patients, ASA classification of patients. In addition, each patient was asked to respond to an information document containing the STOP BANG translated questionnaire by the anesthetist. These patients were referred to the pulmonology department at H.Chaker hospital for ventilatory polygraphy whatever the estimated clinical probability was.

The second phase of the study took place at the pneumology service H.Chaker in Sfax. The patient is then presented in the service where it is equipped with a polygraph device recording his usual bedtime and spends the night in a single room dedicated exclusively to this type recording. The connection and disconnection of the device was made by the doctor.
Each polygraphy has been automatically analysed and must be followed by a manual screening conducted by the sleep specialist. The final interpretation report provides the AHI (apnea hypopnea index).

The severity of OSAS is based on 3 levels: mild $\geq 5 / \mathrm{h}$ and $<$ $15 / \mathrm{h}$, moderate $\geq 15 / \mathrm{h}$ and $<30 / \mathrm{h}$ and severe $\geq 30 / \mathrm{h}$.

The primary outcome measure was the severity of sleep apnea based upon the AHI as categorized into 4 groups: none $(\mathrm{AHI}<$ $5 / \mathrm{h}$ ), mild (AHI $\geq 5$ to $<15 / \mathrm{h}$ ), moderate (AHI $\geq 15$ to $<30 / \mathrm{h}$ ), and severe $(\mathrm{AHI} \geq 30 / \mathrm{h})$. A proportional odds logistic regression analysis was conducted using the total score of the STOP BANG questionnaire as the independent variable with equal weight given to each response (linear model). Coefficient of determination or the proportion of variance was calculated (R2).

\section{Results}

Descriptive summary statistics for the patient population reflected by the STOP-Bang model and the AHI are displayed in Table 1. The mean $\pm \mathrm{SD}$ scores for the STOP-Bang model for males measured 5.2 compared to 3.4 for females. Because male gender is one of the 8 variables, the maximum score for males is 8 , versus 7 for females. The distributions of the STOP-Bang summary scores for the entire group, males, and females are shown in Figure 1. The correlation of the "STOP BANG" questionnaire and the severity of OSAS by the AHI score was R2 $=0.373$. The number of patients in each category of sleep apnea severity (AHI) versus the STOP-Bang model scores is shown in Table 2. With each incremental increase in the score from 0 to 3 , with a significant correlation $(\mathrm{R} 2=0.373$ ) the probability of having no sleep apnea diminished, while the probability of having mild, moderate, or severe sleep apnea increased. With any score $>3$, the probability continuously increased for having severe sleep apnea, while the probability for anything else decreased.

\section{Discussions}

This study confirms that in a surgical population referred for evaluation of sleep apnea disorder, the STOP BANG[6] can be used to estimate the probabilities of no, mild, moderate, and severe obstructive sleep apnea as defined by the AHI. As opposed to dichotomizing a continuous variable in which there is no distinction between a STOP BANG score of 3 and 8, enhanced analysis with the proportional odds logistic regression provides a set

Table 1. Characteristics of the Overall Study Population.

\begin{tabular}{|c|c|c|c|c|c|c|}
\hline & Total & Screened & $\mathbf{A H I}<\mathbf{5 / H}$ & $\mathbf{5} \leq \mathbf{A H I}<\mathbf{1 5} / \mathbf{H}$ & $\mathbf{1 5} \leq \mathbf{A H I}<\mathbf{3 0} / \mathbf{H}$ & $\mathbf{A H I} \geq \mathbf{3 0} / \mathbf{H}$ \\
\hline Number & 759 & 150 & 23 & 35 & 43 & 49 \\
\hline Sex $(\mathrm{M} / \mathrm{F})$ & $453 / 306$ & $85 / 65$ & $8 / 15$ & $20 / 15$ & $27 / 16$ & $30 / 19$ \\
\hline Middle age (years) & $49(18-87)$ & $51(18-87)$ & $54.5(18-52)$ & $56.4(18-87)$ & $55.05(18-81)$ & $67(33-87)$ \\
\hline Middle BMI $\left(\mathrm{kg} / \mathrm{m}^{2}\right)$ & 29.47 & 32.09 & 29.80 & 32.10 & 32.95 & 34.75 \\
\hline BMI $\geq 35\left(\mathrm{~kg} / \mathrm{m}^{2}\right)$ & 42 & 34 & 2 & 7 & 6 & 19 \\
\hline Hypertension & 204 & 64 & 7 & 13 & 15 & 29 \\
\hline Diabetes & 71 & 31 & 10 & 9 & 7 & 4 \\
\hline Tobacco & 162 & 54 & 4 & 11 & & 29 \\
\hline
\end{tabular}


Figure 1. Distribution of affirmative STOP BANG responses.

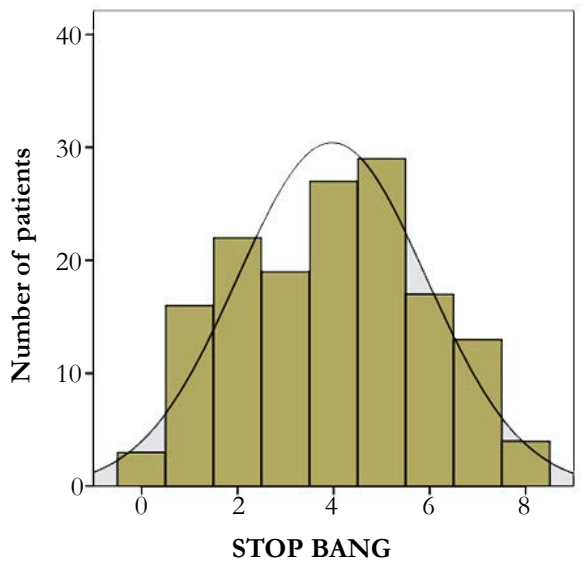

Table 2. Number of Patients with Sleep Apnea Vs. Positive Stop Bang Questionnaire.

\begin{tabular}{|c|c|c|c|c|}
\hline STOP BANG & \multicolumn{5}{|c|}{ Severity of OSAS } \\
\hline & None & Mild & Moderate & Severe \\
\hline 0 & 2 & 1 & 0 & 0 \\
\hline 1 & 4 & 6 & 3 & 3 \\
\hline 2 & 7 & 5 & 6 & 4 \\
\hline 3 & 3 & 6 & 7 & 3 \\
\hline 4 & 2 & 7 & 10 & 8 \\
\hline 5 & 2 & 5 & 8 & 14 \\
\hline 6 & 2 & 2 & 4 & 9 \\
\hline 7 & 1 & 2 & 4 & 6 \\
\hline 8 & 0 & 1 & 1 & 2 \\
\hline
\end{tabular}

of probabilities for various levels of severity. Although intuitive, this study indicates that the greater a single cumulative score of known risk factors as reflected by the STOP BANG questionnaire, the greater the probability of more severe sleep apnea. Of the various models to screen for OSAS, the STOP-Bang model has the appeal of being very straightforward and requires no more than a few minutes to complete and score. Chung et al., [8] reported a sensitivity of $83.6 \%$ for $\mathrm{AHI}>5 / \mathrm{hr}$, our data shows that there is $90.8 \%$ probability of having an $\mathrm{AHI} \geq 5 / \mathrm{hr}$ if the score ranges from 3 to 8 . Therefore, if the only intent is to exclude patients who may have any degree of OSAS from undergoing surgery, then the "STOP BANG" questionnaire appears to be a valid initial screening device. To the extent that the AHI is a valid parameter for defining this complex syndrome, the composite STOP-Bang model score provides a method for stratifying patients into categories of sleep apnea. For example, if the "STOP BANG" score is 6 , then the corresponding probability of having severe OSAS is quite high $(49.4 \%, 71.1 \%$, and $83 \%$, respectively), and further evaluation is relatively urgent. If the score is 3-5, then the probabilities of mild, moderate, and severe OSA are more evenly balanced. Greater discrimination between cases of no, mild, and moderate sleep apnea may not be possible due to the intrinsic deficiencies of the "STOP BANG" questionnaire and limitations imposed by defining sever item of OSAS by only the AHI. The presence of clinically significant sleep disordered breathing found in patients with a composite score of $0-2$ suggests that the number of false negatives may be substantial and that there are other risk factors not identified by this questionnaire. Note, however, that there were relatively few subjects in these low-risk categories, probably because specific population tested consisted predominantly of patients referred for OSAS. In our own study, we showed that there is a positive correlation between the ordinal scales of the "STOP BANG" questionnaire score with the AHI. A degree of underlying uncertainty regarding the probabilities distribution may be created because each point of the STOP Bang score was given equal weight. In fact, our data indicate that a weighted model for each response improves prediction over the linear model. With additional data, it will be easier to detect differences in the effects of the STOP BANG predictors so that models with better information may be derived. The "STOP BANG" questionnaire also does not include other risk factors, such as craniofacial morphology, and comorbidities such as diabetes, coronary artery disease, and stroke [9]. Lastly, these findings may be criticized because we studied a surgical population referred for evaluation of sleep disorders. The statistical method used (proportional odds logistic regression) gives greater information than a yes/no risk determination. Whether or not the probabilities observed in this study apply exactly to other clinic or general populations is not known, but the concept of increasing risk factors being associated with greater likelihood for the presence of more severe sleep apnea is intuitive and seems reasonable. It is implausible that a SBM score of 7-8 in any population would be less predictive of severe OSA; however, the accuracy of discriminating no, mild, and moderate disease in a less selective population with a lower prevalence of OSA is unknown and the validity and application of these results in a more general or different clinical population must be established. The STOP-Bang questionnaire has already been successfully applied in preopera- 
tive screening [10]. The stratification of patients based on "STOP BANG" questionnaire score is interesting to indicate the patient with must have an urgent sleep disorder test [11].

\section{Conclusion}

The STOP BANG questionnaire provides a simple method for screening, estimating the AHI severity, and triaging patients for testing. Using the SBM to provide sets of probabilities may provide greater guidance to clinical decision making than a dichotomous analysis. This concept needs to be verified in a larger clinical trial.

\section{References}

[1]. AASM (1999) Sleep-related breathing disorders in adults: recommendations for syndrome definition and measurement techniques in clinical research. The Report of an American Academy of Sleep Medicine Task Force. Sleep. 22(5): 667-689.

[2]. Vijayan VK (2012) Morbidities associated with obstructive sleep apnea. Expert Rev Respir Med. 6(5): 557-566. doi:10.1586/ers.12.44.

[3]. Peterson GN, Domino KB, Caplan RA, Posner KL, Lee LA, et al., (2005) Management of the difficult airway: a closed claims analysis. Anesthesiology. 103(1): 33-39.
[4]. Kurtipek O, Isik B, Arslan M, Unal Y, Kizil Y, et al., (2012) A study to investigate the relationship between difficult intubation and prediction criterion of difficult intubation in patients with obstructive sleep apnea syndrome. J Res Med Sci. 17(7): 615-620.

[5]. Chung SA, Yuan H, Chung F (2008) A Systemic Review of Obstructive Sleep Apnea and Its Implications for Anesthesiologists. Anesth Analg. 107(5): 1543-1563. doi:10.1213/ane.0b013e318187c83a.

[6]. Chung F, Yegneswaran B, Liao P, Chung SA, Islam S, et al., (2008) STOP Questionnaire: a tool to screen patients for obstructive sleep apnea. Anesthesiology. 108(5): 812-821. doi:10.1097/ALN.0b013e31816d83e4.

[7]. Semelka M, Wilson J, Floyd R (2016) Diagnosis and Treatment of Obstructive Sleep Apnea in Adults. Am Fam Physician. 94(5): 355-360.

[8]. Ramachandran SK, Josephs LA (2009) A Meta-analysis of Clinical Screening Tests for Obstructive Sleep Apnea. Anesthesiology. 110(4): 928-939. doi:10.1097/ALN.0b013e31819c47b6.

[9]. Nahapetian R, Silva GE, Vana KD, Parthasarathy S, Quan SF (2016) Weighted STOP-Bang and screening for sleep-disordered breathing. Sleep Breath. 20(2): 597-603. doi:10.1007/s11325-015-1255-2.

[10]. Vasu TS, Doghramji K, Cavallazzi R, Grewal R, Hirani A, et al., (2010) Obstructive Sleep Apnea Syndrome and Postoperative Complications. Arch Otolaryngol Neck Surg. 136(10): 1020. doi:10.1001/archoto.2010.1020.

[11]. Farney RJ, Walker BS, Farney RM, Snow GL, Walker JM (2011) The STOP-Bang Equivalent Model and Prediction of Severity of Obstructive Sleep Apnea: Relation to Polysomnographic Measurements of the Apnea/ Hypopnea Index. J Clin Sleep Med. 7(5): 459-465. doi:10.5664/jcsm.1306. 